\title{
A Research on the Radar Anti-jamming Evaluation Index System
}

\author{
Wang Feng, Liu Dongsheng, Liu Pengyuan, Li Bing \\ wangfeng1111@126.com \\ Department of Missile Engineering Ordnance Engineering College, Shijiazhuang, 050003 China
}

\begin{abstract}
Keyword: Radar Anti-jamming, Evaluation, Index system
\end{abstract}
\begin{abstract}
The comprehensive evaluation of radar anti-jamming effectiveness is a multi-index comprehensive evaluation problem. Anti-jamming effectiveness evaluation index system is the important and difficult in the process of evaluation. The established index system should be able to comprehensively and objectively reflect the whole anti-jamming performance of radar. The index should be measurability. Selection of radar anti-jamming performance evaluation indicators must be conducted according to the principle of multi-level and various. Construct a complete assessment system and systematically reflect the actual performance of the equipment.
\end{abstract}

\section{Introduction}

Modern electronic warfare equipment presents a highly integrated, smart and intelligent development trend with the rapid developing of electronic warfare technology. Producing high strength, variety and targeted electronic jamming is easy in the whole airspace, the whole frequency domain and time domain. Seriously affect the air defense intelligence radar detection ability. In order to make the radar play more combat effectiveness in a complex interference environment, it is necessary to evaluate the radar anti-jamming system deeply. How to objectively and comprehensively evaluate the anti-interference ability of modern radar systems has become a radar department of design, production and use of important topics of common interest[1].

Radar confrontation process is a process of incomplete information dynamic game, which contains a large number of uncertain factors, human factors and complicated technology. It is very difficult to evaluate the effect and effectiveness[2]. Radar anti-jamming effectiveness of comprehensive evaluation is a multi-index comprehensive evaluation problem. Anti-jamming effectiveness evaluation index system is the important and difficult in the process of evaluation. The established index system should be able to comprehensively and objectively reflect the whole anti-jamming performance of radar. The index should be measurability. Current research of radar anti-jamming effectiveness evaluation index has made some achievements. But have not a unified evaluation standard, evaluation index more and complex as well as the evaluation index is difficult to measure and so on many problems, which affected the objective evaluation of radar anti-jamming effectiveness seriously.

\section{Radar anti-jamming evaluation index system}

\section{The selection principles of evaluation index}

For the selection of radar anti-jamming performance evaluation indicators, selection should be carried out in accordance with the principle of multi-level and multi-dimensional. To construct a complete assessment system, systematically reflect the actual performance of the equipment. During the Index selection process, too much evaluation index not only increased the complexity of the result, also may affect the objectivity of evaluation. The cross should be reduced between evaluation indexes As far as possible, each other should be independent and easy to understand and measure.

To establish evaluation index system, the following principles should be considered:

(1) Indicators of completeness. Assessment system can be effectively described anti-jamming performance of radar.

(2) The clarity of the indicators. The meaning of assessment indicators must be accurate. 
(3) The independence of the indicators. It should be no overlap between evaluation index, and even irrelevant.

(4) Measurability of index. Evaluation index on the concrete realization are operational.

(5) The availability of indicators. Evaluation index should be easy to treat as soon as possible.

(6) Simply of Indicators. Evaluation index difference between primary and secondary, avoid cumbersome.

(7) The hierarchy of indicators. Combat situation is complexity and the combat missions are multiplicity. Jamming performance evaluation is often can not only select a single evaluation index. It is necessary to select multiple level and variety indexes. Form an evaluation index system.

\section{The establishment of evaluation index system}

Now research is mainly on power criterion, tactical application rules, information criterion, probability criterion, etc. In addition, there are other indexes which includes ECCM Improvement Factor, suppression coefficient, information entropy under the condition of interference, Accuracy of measurement, Anti-interference deceive the success rate, detection probability[3]. The above index has realized the radar anti-jamming performance from the conversion of qualitative evaluation to quantitative evaluation at different angles. But some index is difficult to actual measure such as information entropy under the condition of interference and detection probability. As Inherent part of radar, some other index can not be operated independently such as low side lobe of radar antenna, pulse compression and wide - limit-narrow. So the radar should be test in accordance with the active jamming technology. The test evaluation should be performed in accordance with the requirements of practical tactics. In another words, the test should be carried on the actual combat process between radar and jammer. Through the analysis of radar anti-jamming during active disturbance of the tactical performance under the condition of no interference with radar tactical performance difference, reflect the radar anti-jamming performance objectively and truly according to the tactical mission and task requirements.

Radar anti-jamming performance mainly depends on two aspects: radar anti-jamming performance mainly depends on two aspects. The inherent properties of radar are the basic condition of complete function of radar design. It includes the average transmission power, the antenna gain, signal bandwidth, signal wide bandwidth product, work system, etc. The radar working system determines the technical features of the radar. The radar signal bandwidth determines the range resolution. These indicators of radar anti-jamming performance are very important. From the qualitative point of view, radar EIF reflects the improve degree of the signal to jamming after taking various anti-interference measures. Self-defense distance and detection probability reflects the various pressing type interference conditions, radar detection range and the decrease of detection probability. Radar anti-jamming false target jamming success rate, success rate of drawing-out interference, the tracking error represents deception jamming resistance and the deviation of attack range of weapon system in different deception jamming[3]. Radar anti-jamming effectiveness factors including EIF, self-defense distance and effective jamming sectors, deception jamming success rate of resistance, resistance to fractional success rate, detection probability, tracking error, etc. In addition, the anti-interference ability of the radar also reflected on the terminal display. Measure the pros and cons of radar anti-jamming capability by measuring the target signal interference of the sector, target track, the average time to improve the initial improvement factor, system factor, the average false track average number of missing track improvement factor index. So draw lessons from the ideas of the analytic hierarchy process (ahp), combined with the actual radar anti-jamming performance evaluation, the performance index related to the anti-jamming performance of layered processing.

\section{The evaluation index under the condition of tactical}

Expectations of radar jamming effect is to reduce the work performance and unable to complete a given task. We are concerned about its ability to work under a given interference environment. To establish the principle of evaluation index is that normal work is effectively in a given interference 
environment. P represents the performance of radar. Under the tactical condition radar finishing the task need to be able to allow it to take the minimum is Pmin. Then:

$P-P_{\min } \geq 0$ Anti-jamming is effective.

$P-P_{\min }<0$ Anti-jamming is ineffective.

The basic function of search radar is to find and provide an early warning target[4]. Another important task is to provide the following tracking radar target designation and lead it to capture target. Search radar must first find target and determine its position to the measured target parameter passed to the tracking radar. After receiving target designation from search radar, tracking radar search in a certain range. The tracking radar turn to track the status after detected the goal of instruction. Finding the goal and certain target location are the two basic functions search radar. Radar detection performance can be separately prescribed by meet the false-alarm probability of detection probability of the largest found distance measure and measure the performance of the target parameter by the error of measurement. Evaluation of the whole radar system effectiveness should be treated as a gauge. Definition of radar detection ability of anti-jamming coefficient:

$$
C_{R}= \begin{cases}\left(R_{j}-R_{\text {min }}\right) /\left(R_{0}-R_{\text {min }}\right) & R_{j} \geq R_{\text {min }} \\ 0 & R_{j}<R_{\text {min }}\end{cases}
$$

$\mathrm{R}_{0}$ is the radar maximum range without interference. $R_{j}$ is the radar maximum range with interference. $\mathrm{R}_{\min }$ is the minimum self-defense distance which satisfies search radar. The ranging precision of radar anti-jamming coefficient:

$$
C_{\Delta R}= \begin{cases}\left(\Delta R_{\max }-\Delta R_{j}\right) /\left(\Delta R_{\max }-\Delta R_{0}\right) & \Delta R_{j} \leq \Delta R_{\max } \\ 0 & \Delta R_{j}>\Delta R_{\max }\end{cases}
$$

$\triangle R_{0}$ is the radar ranging error without interference. $\triangle R_{j}$ is the radar ranging error with interference. $\triangle R_{\max }$ is the maximum distance measurement error which satisfies tracking radar. Similarly, define the Angle measuring precision of the radar anti-jamming coefficient. Work and measure link detection, so the total radar anti-jamming coefficient effectively:

$$
C=C_{R} C_{\Delta R} C_{\Delta \theta}
$$

Find from above type, If there is an indicator did not meet the minimum requirements of weapon system of the whole radar system anti-jamming coefficient is 0 .

\section{The application in the assessment}

Now, the method of evaluation of radar anti-jamming effect has analytical calculation method, simulation method and experimental method. Test method is to obtain a lot of experimental data based on real radar system by laboratory or field experiment. Then analyze the data to obtain treatment process of the evaluation conclusion[4]. According to the interference environment, the background environment and target echo form the different way, test method is divided into the physical test method and simulation test method. Combined with radar power, precision test method for measuring range is mature in the corresponding interference conditions for evaluating the required data.

The function simulation method of radar uses only the functionality of the radar. For the details contained in the waveform and signal processor is not involved. Deal with it as a system loss. This method is easier and widely used because system-level attack-defense simulation applicability is stronger.

Usually, the radar anti-jamming measures can be divided into frequency selection, signal processing, power confrontation, waveform selection, choice of polarization, space and active interference[5]. Such as wide - limited - narrow anti-jamming circuit, constant false alarm processing (CFAR) and so on. These improve the performance of these against interference in the functional simulation in different measures can be measured by the Signal to jamming ratio. It is reflected in anti-jamming filter module during the simulation. Use functional simulation method to get the radar in interference under the condition of satisfying the false-alarm probability and 
detection probability of the range and accuracy of measurement. According to the requirements of the whole weapon system to search radar performance can get the radar anti-jamming effective coefficient under specific interference.

\section{Conclusion}

Evaluation of radar anti-jamming effectiveness is always a uncertain technical problems for a long time, it is not a unified comprehensive measures and methods yet. This is due to the complexity of the anti-jamming evaluation. The anti-jamming performance of radar system and radar not only relate to its own technical characteristics, and related to operational use of electromagnetic environment, tactical application. Based on the result criterion, this paper puts forward effective coefficient which can measure of anti-jamming tactical request search radar anti-jamming capability well. The research provides a certain reference for radar equipment argumentation and using.

\section{References}

[1] Zhang Youwei, Li Shaohong. Radar system analysis[M]. Beijing: National Defense Industry Press, 1981.

[2] Elbirt A J. Information warfare: are you at risk[J]. IEEE Technology and Society Magazine, 2003, 22(4): 13-19.

[3] Li Shuhua, Huang Xiaogang, Liu ping. Study on anti-jamming technology under complex electronic magnetic enviro-ment[J]. Modern Radar, 2013, 35(4): 6-10, 14.

[4] Liu Xiaodong. Research on radar ECCM effectiveness evaluation index and method[D]. Chengdu: University of Electronic Science and Technology of China, 2008.

[5] Schleher D C. Electronic warfare in the information age[M]. Boston: Artech House, 1999. 